\title{
Estrategias para la convivencia de I.E.D. Rural Palermo ${ }^{1}$
}

\section{Strategies for the coexistence of I.E.D. Rural Palermo}

DOI: http://dx.doi.org/10.17981/cultedusoc.9.2.2018.08

Fecha de recepción: 01/06/2018. Fecha de aceptación: 28/08/2018

\author{
Mabel Salas ${ }^{2}$ \\ Xiomara Barbosa; Clarena Cabrera; Ildefonso González; \\ Humberto Martínez; Alexander Montero; Maryoris Pacheco; \\ Sandra Piña; Amanda Salgado; Deisy Cueto; Denys Mejia; \\ Elsy Castro; Jasmina De La Valle; Carmen Cervantes; \\ Danilza Segura; Inírida Gastelbondo; Lexy Retamozo; \\ Nelsy De La Cruz; Yolanda Peña; Yomaira Fontalvo; Liliana Borja; \\ Amparo Cervantes; Eliana Pedroza y Gloria Morrón \\ IED Palermo (Colombia) \\ augmedimata@hotmail.com
}

Para citar este artículo

Salas, M., Barbosa, X., Cabrera, C., González, I., Martínez, H., Montero, A., Pacheco, M., Piña, S., Salgado, A., Cueto, D., Mejia, D., Castro, E., De La Valle, J., Cervantes, C., Segura, D., Gastelbondo, I., Retamozo, L., De La Cruz, N., Peña, Y., Fontalvo, Y., Borja, L., Cervantes, A., Pedroza, E. y Morrón, G. (2018). Estrategias para la convivencia de I.E.D. Rural Palermo. Cultura. Educación y Sociedad 9(2), 86-94. DOI: http://dx.doi. org/10.17981/cultedusoc.9.2.2018.08

\section{Resumen}

La presente investigación tuvo como objetivo reconstruir la historia del corregimiento de Tasajera para la preservación de la identidad cultural de los estudiantes. La investigación es de tipo cualitativa, la población participante son los estudiantes de cuarto grado de la Institución Educativa Rural Palermo. Como técnica de recolección de información se utilizó la entrevista y como instrumento se aplicó la historia de vida. Como conclusiones es importante inferir que a interior de la escuela existe poca tolerancia entre los estudiantes por lo que el reto, para la Institución Educativa es realizar estrategias que fortalezcan una sana convivencia de la mano con la libertad y la forma de pensar de la comunidad, debido a que existe una analogía con la educación, por lo que éstos factores benefician a las personas en situaciones problemáticas frecuentes en su cotidianidad.

Palabras clave: Reconstrucción, preservación, identidad cultural.

\begin{abstract}
The objective of the present investigation was to reconstruct the history of the corregimiento of Tasajera for the preservation of the cultural identity of the students. The research is qualitative, the participating population is the fourth grade students of the Palermo Rural Educational Institution. As an information gathering technique, the interview was used and the history of life was applied as an instrument. As conclusions it is important to infer that within the school there is little tolerance among students so the challenge, for the Educational Institution is to implement strategies that strengthen a healthy coexistence hand in hand with democracy and citizenship training since it has a close relationship with education and therefore these aspects favor individuals so that they have and can carry in the situations that are presented to them on a daily basis.
\end{abstract}

Keywords: Reconstruction, preservation, cultural identity.

\footnotetext{
${ }^{1}$ Este artículo ha sido derivado del Programa de Fortalecimiento de la Cultura Ciudadana y Democrática CT+I a través de la IEP apoyada en TIC en el Departamento de Magdalena: CICLÓN. Desarrollado con docentes miembros del Grupo de investigación "Investigadores Palermistas en acción" pertenecientes a la Institución Educativa Departamental Palermo.

${ }^{2}$ Líder de investigación del grupo "Investigadores Palermistas en acción".
}

- The author; licensee Universidad de la Costa - CUC. 


\section{Introducción}

La presente investigación se base en tres pilares fundamentales: Valores para la convivencia, la convivencia escolar y las áreas de gestión. Para que haya una buena convivencia escolar hay que elegir acciones entre todos aquellos que hacen parte de la comunidad educativa, tanto directivos, padres de familia y estudiantes darán su opinión, de ésta forma pondremos en práctica valores que contribuyan en la formación ciudadana.

"La educación es el proceso que le permite al estudiante el reconocimiento de sí mismo, de sus sentimientos, actitudes, aptitudes, valores y emociones, lo cual se ve en las relaciones que entabla consigo mismo y con los demás, en el contexto en el que se desenvuelve. Esto hace referencia a la formación del individuo pues, más que nada, no simplemente se enfoca en la práctica de valores para una convivencia sana, sino también en formar a los individuos de una u otra manera para que sean competentes en la sociedad en general, así como también con el fortalecimiento de sus virtudes" (Agudelo C, 1998) ; es decir, como proceso complejo, es vista desde una perspectiva multidimensional, integradora para formar el ciudadano que requiere la sociedad actual capaz de evaluar y autoevaluarse para asumir una postura crítica frente a su rol en su entorno (Soto y Martínez, 2017).

La democracia y la formación ciudadana poseen una analogía con la educación, por lo que éstos factores benefician a las personas en situaciones problemáticas frecuentes en su cotidianidad dentro de los establecimientos educativos, dichos factores favorecerán no solos los estudiantes, sino a todos aquellos que hace parte de la comunidad educativa. (Gonzalez-Luna, 2010) manifiesta que la analogía entre educación, ciudadanía y democracia es precisa, y se muestra de modo específico en la sociedad a través del tiempo. Ratificando lo dicho anteriormente por los autores, para que haya una buena convivencia, los aspectos que una persona debe tener en cuenta para mejorar son diversos previamente a conseguir buenos lazos con sus semejantes en el contexto que lo rodea.

Si se dese tener una buena convivencia necesariamente se requiere de una buena formación, la cual se empieza a formar en el hogar. Ponemos en práctica una buena convivencia en las instituciones educativas cuando el estudiante establece una agradable relación con sus maestros, compañeros y las demás personas que hacen parte de la institución. Tomando como referencia la convivencia de la mayoría de las instituciones educativas nos daremos cuenta que es pésima, para que se normalice y mejore, debe primar la condición de que los alumnos interactúen más entre ellos y que ellos mismo participen en la elaboración de normas. "El alumnado tiene derecho a intervenir en la elaboración y aplicación de las normas de convivencia, no sólo como un colectivo que puede aportar muchos elementos de mejora y reflexión sino porque la intervención en el proceso de elaboración se convierte en un aprendizaje que permite actuar como ciudadanos responsables dentro y fuera del centro" (Noto, 2002).

Consideramos que los estudiantes realizan activamente los ejercicios que se formulan para mejorar la convivencia, porque de una u otra forma empezarían a practicar la falta de valores, y se podrá ver si cada uno de ellos son responsables, como lo indica (Viñas, 2002) "La participación es un objetivo educativo, una forma de organizar el centro desde la corresponsabilidad. No es un proceso lineal". 
La comunicación resulta necesaria para poder realizar actividades que se relacionas con la solución de problemas, con el compromiso de los maestros y visión y misión de la institución. Dicha intervención para buscar una solución a los conflictos se resume a cuatro niveles (Viñas, 2002), lo anteriormente mencionado, se piensa que los individuos que no poseen una excelente educación o que no fueron educados de la manera que se cree correcta, no alcanzan a ser sociables y a tener una buena relación con aquellas personas con las que interactúan en su contexto, son varios los factores que influyen para que haya una buena convivencia, una de las cuales es que los individuos que no tuvieron una buena educación no alcanzan a dar solución a los problemas que surgen cuando hay una pésima convivencia.

\section{Valores para la convivencia}

Los valores no son creación del hombre, pero es quien los descubre, y al hacerlo los hace suyo. Así ostentan una llamativa energía, la cual releja la apreciación de las personas, por ello las personas están en constante descubrimiento de sentido en las cosas que descubre dejando de lado aquellas que ya no tienen el mismo significado para él. La escala de los valores se puede apreciar, es de carácter cualitativo y no cuantitativo, por lo que los valores resultan ser cualidades de los individuos; no hay parámetros objetivos para valorarlos en ningún campo, estar en manos de la situación estimativa de las personas.

Los aspectos que corresponde a la ética y los valores de las personas un lugar de acuerdo a las ciencias exactas: matemáticas, física, química y demás. Con la ley General de Educación (Ley 115 del 94) se pudo lograr transformar el anterior escenario, dando equilibrio a las intenciones principales de la educación, tomando como referencia los aspectos fundamentales de la cultura, entre ellos su extensión ética y humanística.

Ley General de Educación para fundamentar éticamente el rol de las personas en sociedad.

Ley 115 de 1994.

Artículo 5: Fines de la educación

Artículo 13. Objetivos comunes a todos los niveles educativos

Artículo 15. Definición de educación Preescolar. Artículo 20. Objetivos generales de la educación básica.

Artículo 21. Objetivos específicos de la educación básica.

Artículo 23. Áreas obligatorias y fundamentales. Artículo 24. Educación Religiosa.

Artículo 25. Formación ética y moral.

Artículo 30. Objetivos de la educación media y académica.

Artículo 80. Evaluación de la educación.

Artículo 92. Formación del educando.

Artículo 104. El educador.

Artículo 109. Finalidades de la formación de educadores.

Fuente: Elaboración propia. (2018).

Construir convivencia quiere decir colocar acento en el área de educación, desprendiéndose de las situaciones escolares diarias; creyendo que la palabra es lo más propio de las personas, como consecuencia, el entorno que lo rodea es la mejor opción para su desarrollo. Confiar en la palabra significa conseguir espacios propicios para desarrollarla, dando lugar a la participación grupal, con la palabra se expresan emociones, dicha participación puede ser regulada por personas adultas responsables, los cuales deben ser referentes y ejemplos para los niños: adultos que necesitaran su propio espacio para dialogar, hacer propuestas, críticas constructivas y demás.

Estos grupos de participación reglamentan las instituciones educativas creando responsabilidades, lazos y obligaciones. A través de la palabra se puede 
promover el diálogo, la comunicación, la reflexión, el juicio y la confianza. Guerrero (2016) define a los valores como "convicciones profundas de los seres humanos de que algo es preferible y digno de aprecio, que determinan su manera de ser y orientan su conducta”. Dentro de este grupo de valores se destacan el dialogo y la tolerancia, como bases fundamentales para una sana convivencia escolar, con todos los integrantes de la comunidad educativa.

Ortega y Minguez en su libro Los valores de la educación (Ortega \& Minguez, 2001) dedican dos capítulos al diálogo y la tolerancia, aunque se analice por separado son factores fundamentales e intrínsecos para poder alcanzar una buena convivencia.

Para aclarar los conceptos Otega y Minguez encaminan su definición más allá de lo brindado por un diccionario, y encaminan definirlos desde medidas más profundas e incluyentes: "desde el reconocimiento de la igual legitimidad de los interlocutores y la voluntad de comprender y respetar las diferencias, como reconocimiento de la dignidad del otro, como depositario de confianza, reciprocidad y comunión; como mutuo reconocimiento y confianza recíproca; como reconocimiento del otro en su irrenunciable alteridad y diferencia" (Duch, 1997).

Con el diálogo a través de su pluralidad podemos conseguir el respeto, la comprensión y la aceptación de los demás sin restricciones, Ortega y Minguez (Ortega \& Minguez, 2001) impacientes por fundamentar el diálogo, estudiaron varios autores (entre ellos Ortega y Gasset, Buber, Zubiri, Díaz, etc.) cuyos estudios permitieron redimensionar las trascendencias del diálogo más allá de ser el medio para la obtención de resultados de índole diversos. De ahí que los autores lo propongan como un valor en sí mismo, sin negar su carácter pragmático.

Para evolucionar los seres vivos necesitamos aprender y aprehender, señalan los autores, es por ello que también se tiene que aprender a dialogar, por lo cual enumeran unas actitudes: "la actitud de respeto, la búsqueda de la verdad, la no imposición de nuestra verdad, el reconocimiento de la igual dignidad del otro, etcétera. Al igual se requiere de ciertas habilidades, de éstas mencionan la capacidad de empatía y de autocontrol como elementos sustanciales en una comunicación dialógica" (Ortega \& Minguez, 2001). Al momento de afrontar los temas de diálogo y educación, alegan que educación no es precisamente transmitir saberes, y no se educa al momento de transferir un método específico de valores; tomando como referencia a Peters precisan la educación como el acto que "implica comprometerse en la utilización de procedimientos legitimados por la moral" (Ortega \& Minguez, 2001).

La relación que se da en la educación no se refiere a una relación de poder, de reconocimiento o de afirmación contra el otro, por lo que se hace necesario en la educación el diálogo. Los autores Ortega y Minguez a través del humus nos ponen el ejemplo de fértil, de nutriente, por el cual es el componente etimológico de la palabra humildad (del latín humilitas, abajarse; de humus: tierra) y es a la vez (la humildad) como se dijo anteriormente, sustancia y a la vez condición ineludible del diálogo.

Sin embargo, en una sociedad múltiple o "democrática" igual en la que vivimos, donde la intolerancia, discriminatoria y demás están presentes por doquier; por lo que la guía, los cuidados y el acompañamiento por medio del diálogo se hacen necesarias según los autores. 


\section{Convivencia Escolar}

Las instituciones educativas son determinantes en el proceso de edificación de resiliencia, dentro de éstas se ponen en desarrollo los procesos sociales y humanos determinantes para los seres humanos, por lo que agrega un valor importante en la formación académicas de las personas como seres sociales que desarrollan actitudes para residir con los demás y dar solución a los inconvenientes que se presente. La convivencia es básicamente vivir con el otro, aceptar al otro y crear un ambiente en el que la comunicación y el desarrollo personal se pueda dar libremente. Para Martinez-Otero la convivencia es "tanto como referirse a la vida en compañía de otros, la vida humana solo es posible merced a la participación de los demás" (Ortega \& Minguez, 2001). Por otro lado Ruiz define la convivencia como "una suma de varios factores que nos hacen vivir con otros bajo pautas de conducta que permiten la aceptación del otro" (Ruíz, 2007), además la autora afirma, "la convivencia encierra un cierto bien común que es conveniente respetar, lo que favorece la espontanea resolución de conflictos" (Ruíz, 2007).

Asimismo, La Ley sobre Violencia Escolar concibe la buena convivencia "como la coexistencia pacífica de los miembros de la comunidad educativa, que supone una interrelación positiva entre ellos y permite el adecuado cumplimiento de los objetivos educativos en un clima que propicia el desarrollo integral de los estudiantes".

La convivencia escolar se trata de edificar una forma en la que se puedan relacionar las personas de una comunidad, basada en el respeto y en la solidaridad mutua, que se expresa en la interrelación armoniosa y sin violencia entre los diferentes participantes y cuerpos de la Comunidad Educativa que posee un enfoque predominantemente formativo. Se debe instruir y aprender una suma de conocimientos, habilidades y valores que permitan poner en práctica el vivir en paz y armonía con otros, porque es la base para el ejercicio de la ciudadanía. En la escolaridad, estos aprendizajes están establecidos tanto en los Objetivos Fundamentales Transversales como en los Objetivos Fundamentales Verticales.

El enfoque formativo contiene una dimensión preventiva, que se expresa en la medida en que vamos desarrollando habilidades, conocimientos y actitudes que faciliten la formación de personas íntegras e independientes, competentes a la hora de tomar decisiones personales y de pronosticar circunstancias que alteren y pongan en peligro el aprendizaje de la convivencia, previniendo en cualquier instante el proceso de formación y estrategias pedagógicas. De este modo, la dimensión preventiva involucra superar los riesgos y se cohíbe solo de informar y prohibir, apuntando a intervenir con anticipación. Los factores que pueden llegar a afectar la convivencia escolar, según (Tuvilla R, 2004) pueden son "los factores biológicos y de la historia personal que influyen en el comportamiento de una persona. Estos son: impulsividad, bajo nivel educativo, abuso de sustancias psicotrópicas y antecedentes de comportamiento agresivo o de haber sufrido maltrato", etc.

\section{Metodología}

El presente estudio es de tipo cualitativo, con un alcance descriptivo, pues tal como lo expone Hernández, Collado y Batista (2014), se busca comprender e interpretar la realidad de los fenómenos tal como suceden en su contexto natural. 


\section{Escenarios y actores}

Los participantes son los estudiantes de la Institución Educativa Rural Palermo, conformando un grupo de (30) estudiantes del grado cuarto de básica primaria.

\section{Técnicas e instrumentos de recolección de la información}

Los instrumentos aplicados, para el estudio fue la entrevista estructurada y la observación participante, para ser contrastados con los postulados teóricas establecidos (Jiménez, 2012).

\section{Procedimiento}

La ejecución de la presente investigación está enmarcada en los siguientes momentos:

Momento (1): Elección del fenómeno a estudiar.

Momento (2): Hacer el sondeo diagnóstico de los objetos de interés.
Momento (3): Definición de las categorías de estudio: convivencia escolar, estrategias pedagógicas.

Momento (4): Definición de los instrumentos de recolección de la información. Momento (5): Aplicación de la entrevista estructurada y los registros de observación.

Momento (6): Sistematización de la información a la luz de los datos recolectados y articulación con la teoría.

Momento (7): Generación de resultados. Momento (8): Socialización de los hallazgos encontrados.

\section{Resultados}

A continuación, se presentan los datos recolectados por los instrumentos aplicados tomando en cuenta una pregunta orientadora.

¿Cuáles son las estrategias que promueven la convivencia entre los estudiantes de la Institución Educativa Rural Palermo?

\begin{tabular}{|c|c|c|c|}
\hline $\begin{array}{l}\text { Categoria } \\
\text { de estudio }\end{array}$ & $\begin{array}{c}\text { Pregunta } \\
\text { orientadora }\end{array}$ & Discurso de los actores & $\begin{array}{c}\text { Articulación y } \\
\text { sistematización teórica }\end{array}$ \\
\hline $\begin{array}{l}\text { Convivencia } \\
\text { escolar }\end{array}$ & $\begin{array}{l}\text { ¿Qué es la } \\
\text { convivencia } \\
\text { escolar? }\end{array}$ & $\begin{array}{l}\text { "Es cuando nos relacionamos } \\
\text { con los demás", "es cundo } \\
\text { hacemos actividades con ellos", } \\
\text { "cuando trabajamos en grupo", } \\
\text { "cuando hacemos tareas, y } \\
\text { jugamos", "la convivencia es lo } \\
\text { que vivimos en la escuela cono } \\
\text { nuestros amigos", "es cuando } \\
\text { nos ayudamos entre todos", } \\
\text { "la convivencia es la que nos } \\
\text { permite querernos y aceptarnos } \\
\text { como somos, porque todos } \\
\text { tenemos defectos y muchas cosas } \\
\text { buenas" }\end{array}$ & $\begin{array}{l}\text { Los estudiantes participantes } \\
\text { distinguen que convivir implica } \\
\text { a relación con los demás, y esto } \\
\text { es soportado en la definición que } \\
\text { soportan los autores cuando dicen } \\
\text { que la convivencia es básicamente } \\
\text { vivir con el otro, aceptar al otro } \\
\text { y crear un ambiente en el que } \\
\text { la comunicación y el desarrollo } \\
\text { personal se pueda dar libremente. } \\
\text { Para (Martínez-Otero Pérez, 2001) } \\
\text { la convivencia es "tanto como } \\
\text { referirse a la vida en compañía } \\
\text { de otros, la vida humana solo es } \\
\text { posible merced a la participación de } \\
\text { los demás". }\end{array}$ \\
\hline
\end{tabular}




\begin{tabular}{|c|c|c|c|}
\hline $\begin{array}{l}\text { Estrategias } \\
\text { pedagógicas }\end{array}$ & $\begin{array}{l}\text { ¿Tus profesores } \\
\text { hacen } \\
\text { actividades } \\
\text { para mejorar } \\
\text { las relaciones } \\
\text { en el salón de } \\
\text { clases? }\end{array}$ & $\begin{array}{l}\text { "si, hacen juegos donde todos } \\
\text { participamos", "nos dicen } \\
\text { que no peleemos porque en el } \\
\text { colegio somos como hermanos", } \\
\text { "nos hace que nos contentemos } \\
\text { cuando peleamos", "llaman a los } \\
\text { papas porque a cada rato hay } \\
\text { peleas cuando los compañeros } \\
\text { se burlan de los otros, y le } \\
\text { pegan a los otros" "nos colocan } \\
\text { a dialogar cuando discutimos, } \\
\text { y nos dicen que nos pidamos } \\
\text { perdón", "los profesores nos } \\
\text { regañan todo el tiempo cuando } \\
\text { empezamos a pelear, gritar } \\
\text { maltratarnos,, porque dice que } \\
\text { nosotros venimos al colegio es a } \\
\text { aprender". }\end{array}$ & $\begin{array}{l}\text { Son los maestros los que buscan } \\
\text { e ¿incansablemente espacios y } \\
\text { alternativas que fomenten la } \\
\text { sana convivencia, y es el dialogo } \\
\text { una herramienta esencial para } \\
\text { darle solución a cualquier tipo de } \\
\text { inconveniente, así como lo soportan } \\
\text { autores como (Ortega \& Minguez, } \\
\text { 2001) en su procuración por } \\
\text { fundamentar el diálogo, revisan } \\
\text { diversos autores (Ortega y Gasset, } \\
\text { Buber, Zubiri, Díaz, etc.) cuyo } \\
\text { conjunto de reflexiones permiten } \\
\text { redimensionar los alcances del que } \\
\text { tiene expresar de manera armoniosa } \\
\text { los acuerdos y desacuerdos frente a } \\
\text { los comportamiento de los demás, } \\
\text { para la obtención de resultados de } \\
\text { índole diversos. }\end{array}$ \\
\hline $\begin{array}{l}\text { Convivencia } \\
\text { escolar y } \\
\text { estrategias } \\
\text { pedagógicas }\end{array}$ & $\begin{array}{l}\text { ¿Cómo es la } \\
\text { relación con } \\
\text { tus compañeros } \\
\text { buena, regular } \\
\text { o mala? }\end{array}$ & $\begin{array}{l}\text { "a veces peleamos por bobadas", } \\
\text { "es buena por ratos y por otros } \\
\text { peleamos", "tenemos discusiones } \\
\text { porque ellos son muy burlones", } \\
\text { "me peleo con ellos a veces y } \\
\text { luego ya nos hablamos, porque } \\
\text { el profe dice que no debemos } \\
\text { pelear", "yo me hablo con todos, } \\
\text { aunque hay algún compañero } \\
\text { peleonero", "en el salón son muy } \\
\text { burlones y le cogen las cosas a } \\
\text { uno". }\end{array}$ & $\begin{array}{l}\text { Según Quiroz (2011), el acto de } \\
\text { convivir implica un sinnúmero de } \\
\text { cosas, lograr comprender y sobre } \\
\text { todo aceptar los pensamientos y } \\
\text { comportamientos de los demás es } \\
\text { uno de los mayores inconvenientes } \\
\text { que se presentan al interior de las } \\
\text { instituciones educativa pues existe } \\
\text { poca tolerancia en las relaciones, } \\
\text { en el acto de convivir con y esto es } \\
\text { evidenciado en constantes maltratos } \\
\text { fiscos y verbales, bulliyng, entre } \\
\text { otros sucesos. }\end{array}$ \\
\hline
\end{tabular}

Fuente: Elaboración propia. (2018)

\section{Conclusiones}

De acuerdo a los hallazgos encontrados en la entrevista aplicada, se deduce que lo estudiantes no tienen sanas normas de convivencia en el aula, consecuencia de cómo lo dice Quiroz "la educación es el proceso que le permite al estudiante el reconocimiento de sí mismo, de sus sentimientos, actitudes, aptitudes, valores y emociones, lo cual se ve en las relaciones que entabla consigo mismo y con los demás".

Uno de los métodos como anteriormente lo hemos mencionado, para mejorar la convivencia es la democracia y la formación ciudadana, ya que guar- dan una relación con el proceso educativo, consecuentemente éstos factores benefician al individuo ayudándolo a obtener la capacidad de solucionar situaciones de conflicto que se le presente en su diario vivir en su contexto escolar, y no solo con sus compañeros, sino con todos aquellos que hacen parte de la comunidad educativ, así como lo menciona Gonzáles-Luna "las relaciones entre la educación, la ciudadanía y la democracia son estrechas y se manifiestan de manera diferenciada en la historia y en las sociedades". 


\section{Referencias}

Agudelo C, H. A. (1998). Educación en los valores. Bogotá, Colombia. Obtenido de http://www.worldcat.org/title/ educacion-en-los-valores-talleres-pedagogicos/oclc/57073879.

Alonso, L. E. (2007). Sujeto y discurso: el lugar de la entrevista abierta en las prácticas de la sociología educativa. Obtenido de http://mastor.cl/blog/wpcontent/uploads/2016/01/Alonso-Cap2-Sujeto-y-Discurso-El-Lugar-de-LaEntrevista-Abierta.pdf.

Ander-Egg, E. (1977). Técnicas de investigación social (23 ed.). Buenos Aires, Argentina: Magisterio de Río de la Plata. Obtenido de http://abacoenred.com/wp-content/ uploads/2017/05/T\%C3\%A9cnicasde-investigaci\%C $3 \%$ B 3n-socialed.23-Ander-Egg-Ezequiel.pdf.pdf.

Araos San Martín, J. (2008). Relativismo, tolerancia y democracia (Vol. 3). (Veritas, Ed.) Chile. Obtenido de file://C:/Users/HP/Downloads/Dialnet-RelativismoToleranciaYDemocraciaEnHKelsen-2723232.pdf.

Avendaño, I., Cortés, O., Guerrero, H. (2015) Competencias sociales y tecnologías de la información y la comunicación como factores asociados al desempeño en estudiantes de básica primaria con experiencia de desplazamiento forzado Diversitas: Perspectivas en Psicología, vol. 11, núm. 1, 2015, pp. 13-36 Universidad Santo Tomás Bogotá, Colombia.http://www. scielo.org.co/pdf/dpp/v11n1/v11n1a02. pdf.

Casassus, J. (2000). Problemas de la gestión educativa en América Latina. Santiago de Chile, Chile. Obtenido de http://www.lie.upn.mx/docs/Especializacion/Gestion/Lec2\%20.pdf.
Chacón, E. (2011). Gestión institucional para la mediación de conflictos en ambientes de convivencia escolar. $\mathrm{Ob}$ tenido de http://e4c9a13.soopbook.es/.

Duch, L. (1997). La educación y la crisis de la modernidad. España: Paidós. Obtenido de https://www.planetadelibros.com/libro-la-educacion-y-la-crisisde-la-modernidad/18481.

Gimeno, J. (2007). Investigacion e Innovacion sobre la Gestion Pedagogica de los Equipos ensayos y trabajos de investigación. Madrid: Morata. Obtenido de http:/galeon.com/chanram/parte1.pdf.

González-Luna, T. (2010). Democracia y formación ciudadana. Tepepan, México. Obtenido de https://www.ses.unam. $\mathrm{mx} /$ curso2015/pdf/2oct-GonzalezLuna.pdf.

Guerrero, H. y Cepeda, M. (2016) Uso de estrategias pedagógicas para el fortalecimiento de la convivencia escolar de jóvenes vulnerables. Obtenido de http:// saber.ucv.ve/ojs/index.php/rev_ped/article/view/12501.

Hernández, R., Fernández, C., \& Baptista, M. D. (2004). Metodología de la investigación (5 ed.). México: McGraw Hill. Obtenido de https://www. esup.edu.pe/descargas/dep_investigacion/Metodologia\%20de\%20la\%20 investigaci\%C 3\%B3n\%205ta\%20 Edici\%C3\%B3n.pdf.

Hernández, I. (2004). Educar para la tolerancia: una labor conjunta. Revista mexicana de ciencias políticas $y$ sociales. Obtenido de http://www. revistas.unam.mx/index.php/rmcpys/ article/view/42457.

Herrera, B. (2014). Las acciones colectivas en Colombia frente a una realidad global: El derecho de consumo. Barranquilla, Colombia. Obtenido de http://www.scielo.org.co/pdf/just/n25/ n25a04.pdf. 
Soto Salas, H, y Martínez J.C. (2017). La Evaluación : más alla del concepto; noción en los enfoques de evaluación. En, González Velasco J M (coord.) Educación Emergente. Paradigma del Siglo XXI. (pp. 77-89) Bolivia. Editorial PRISA Ltda.

Martínez-Otero Pérez, V. (2001). Convivencia escolar: problemas y soluciones (Vol. 12). Madrid: Educación.

Noto, C. (2002). Normas de convivencia en el aula y en el centro. Barcelona: Mayor. Obtenido de http://aprendeenlinea.udea.edu.co/revistas/index.php/ revistaeyp/article/view/9836/0.

Olaya Villar, M. D. (2002). Educación en valores: La Tolerancia. Albacete, España. Obtenido de https://previa. uclm.es/ab/educacion/ensayos/pdf/revista17/17_12.pdf.

Ortega, P., \& Minguez, R. (2001). Valores de la educación para el desarrollo. Obtenido de file:///C:/Users/HP/Downloads/Dialnet-ValoresYEducacionParaElDesarrollo-117893.pdf.

Peters, R. (1969). El concepto de educación (1 ed.). Buenos aires: Paidós. Obtenido de http://www.bibvirtual.ucb.edu.bo/ opac/Record/144551/Description.

Quiroz, R. E. (2011). Currículo crítico en la formación ciudadana. Medellín: Arbitrados. Obtenido de http://www.saber. ula.ve/bitstream/123456789/35318/1/ articulo6.pdf.

Rodríguez-Burgos, K., Martínez Cárdenas, A. A., \& Rodríguez-Serpa, F. A. (2017). Estudio empírico sobre los valores democráticos de tolerancia y respeto en la generación milenaria. Barranquilla, Colombia. Obtenido de http://www. scielo.org.co/scielo.php?pid=S0124$74412017000100135 \&$ script $=$ sci abstract\&tlng $=$ es.
Ruíz, R. O. (2007). La convivencia: un regalo dela cultura a la escuela. Córdoba, España. Obtenido de https://www.researchgate. net/publication/39219654_La_ convivencia_un_regalo_de_la_ cultura_a_la_escuela.

Tejedor, F. J. (2004). Competencias de los profesores para el uso de las TIC en la enseñanza. Análisis de sus conocimientos y actitudes. (R. e. pedagogía, Ed.) Salamanca, España. Obtenido de file:///C:/Users/HP/ Downloads/Dialnet-CompetenciasDeLosProfesoresParaElUsoDeLasTICEnLaEn-1973261.pdf.

Tuvilla R, J. (2004). Convivencia escolar y resolución pacífica de los conflictos. Barcelona, España. Obtenido de http://soda.ustadistancia.edu.co/ enlinea/pazatiempo/eje3/mod5/unidad1/Resolucion_pacifica_conflictos.pdf.

Viñas, J. (2002). Comunicación y participación en el centro y en el aula para la resolucion de conflictos. Barcelona, España. Obtenido de https:// books.google.com.co/books?id=eT-dT LzaGAEC\&pg=PA39\&lpg=PA39\&d $\mathrm{q}=.+$ Comunicaci\%C3\%B3n+y+parti cipaci $\% \mathrm{C} 3 \% \mathrm{~B} 3 \mathrm{n}+\mathrm{en}+\mathrm{el}+$ centro+y+e $\mathrm{n}+\mathrm{el}+$ aula+para+la+resoluci\% $\mathrm{C} 3 \% \mathrm{~B}$ $3 n+d e+$ conflictos.\&source $=b l \& o t s=u$ WIGZqwFp3\&sig=7LJzNDvtJ9UL CmxIlZ1eXzg1Aq0\&hl=es\&sa $=X \& v$ ed=0ahUKEw. 